\title{
A New Dempster-Shafer Theory-based Method with Fuzzy Targets for Fuzzy Sets Ranking
}

\author{
${ }^{1}$ Kok Chin Chai, ${ }^{1 *}$ Kai Meng Tay, ${ }^{2}$ Chee Peng Lim \\ ${ }^{1}$ Universiti Malaysia Sarawak, Kota Samarahan, Sarawak, Malaysia \\ ${ }^{2}$ Centre for Intelligent Systems Research, Deakin University, Australia. \\ E-mail:*kmtay@feng.unimas.my
}

\begin{abstract}
In this paper, a new Fuzzy Set (FS) ranking method (for type-1 and interval type-2 FSs), which is based on the Dempster-Shafer Theory (DST) of evidence with fuzzy targets, is investigated. Fuzzy targets are adopted to reflect human viewpoints on fuzzy ranking. Two important measures in DST, i.e., the belief and plausibility measures, are used to rank FSs. The proposed approach is evaluated with several benchmark examples. The use of the belief and plausibility measures in fuzzy ranking are discussed and compared. We further analyze the capability of the proposed approach in fulfilling six reasonable fuzzy ordering properties as discussed in [9]-[11].
\end{abstract}

Keywords- Dempster-Shafer theory(DST) of evidence, fuzzy ranking, fuzzy targets, theoretical properties

\section{INTRODUCTION}

Consider $n$ Fuzzy Sets (FSs), denoted as $A_{1}, A_{2}, \ldots, A_{n}$. Fuzzy ranking is a procedure that attempts to compare and order these $n$ FSs in an ascending or descending sequence. The importance of fuzzy ranking has been highlighted in a variety of application domains, e.g., decision-making [1][2], data analysis [3] and risk assessment [4]. Indeed, many fuzzy ranking methods [1]-[11], either for Type-1 (T1) and/or Interval Type-2 (IT2) FSs are available. However, it is yet to have a method that can always give a satisfactory solution in every situation [5][6]; hence further research is necessary.

A search in the recent literature reveals that several new fuzzy ranking methods [6]-[9], which are based on complicated mathematical principles, have been proposed. As an example, an interval and FS comparison method with probability and the Dempster-Shafer Theory (DST) was proposed in [7]. A probability-based ranking approach with fuzzy targets [1][6][8] was presented in [6]. The proposed approach in [6] is limited to T1FSs. It also is unable to distinguish the ranking order of T1FSs under certain conditions [8]. For these reasons, a likelihood-based approach with fuzzy targets was introduced [8]. $\mathrm{Wu}$ and Mendel also presented a fuzzy ranking method that is based on the centroid of IT2FSs [9]. They suggested that an ideal fuzzy ranking method should comply with six reasonable ordering properties [9]-[11]. However, their method cannot always satisfy two of the properties [9]. Even though the importance of these six reasonable ordering properties is known, their fulfillment is not discussed in [6]-[8].

In this paper, we propose a new method, which is a synthesis of DST of evidence with probabilistic interpretation [7][12] and fuzzy targets, for ranking T1FSs and IT2FSs. It is worth mentioning that the work in [7] uses DST for fuzzy ranking of two T1FSs. However, it is not clear how the proposed approach can be extended to fuzzy ranking of more than two FSs and/or IT2FSs. Comparing with the work in [7], our proposed approach allows more than two FSs to be ranked simultaneously. Fuzzy targets [1][6][8] are adopted to reflect human viewpoints on fuzzy ranking. In this paper, two important measures in DST, i.e., the belief and plausibility measures, are used to rank fuzzy sets. We focus on the ranking of triangular and trapezoidal FSs. To evaluate our proposed approach, examples from [8][9] are used. The fulfillment of six reasonable ordering properties [9]-[11] by the proposed approach is further discussed.

The rest of the paper is organized as follows. In section II, some background information on TIFSs and IT2FSs is presented. Section III presents a review on DST of evidence with its probabilistic interpretation. Section IV presents the new fuzzy ranking method based on DST of evidence with fuzzy targets. In section $\mathrm{V}$, we present some examples to illustrate our proposed method in T1FSs and IT2FSs. Finally, concluding remarks are presented in section VI.

\section{BACKGROUND}

\section{A. Definitions}

In this section, some notations related to T1FS and IT2FS are defined.

Definition 1: The $\alpha_{j}$-cut of an T1FS, i.e., $A_{i}$, is denoted by $A_{i}\left(\alpha_{j}\right)$, where $A_{i}\left(\alpha_{j}\right)=\left[a_{i l}\left(\alpha_{j}\right), a_{i r}\left(\alpha_{j}\right)\right], 0 \leq \alpha_{j} \leq 1$, $j=1, \ldots, m$ and $i=1, \ldots, n$. Note that $a_{i l}\left(\alpha_{j}\right)$ and $a_{i r}\left(\alpha_{j}\right)$ denote the left and right endpoints of the $\alpha_{j}$-cut for $A_{i}$, respectively.

Definition 2: A trapezoidal FS is depicted in Fig. 1, where $\left[a_{i 1}, a_{i 2}, a_{i 3}, a_{i 4} ; h\left(A_{i}\right)\right]$ represents the parameters of the corresponding Membership Function (MF) and the height of the T1FS of $A_{i}$, respectively. When $a_{i 2}=a_{i 3}$, it is a triangular T1FS.

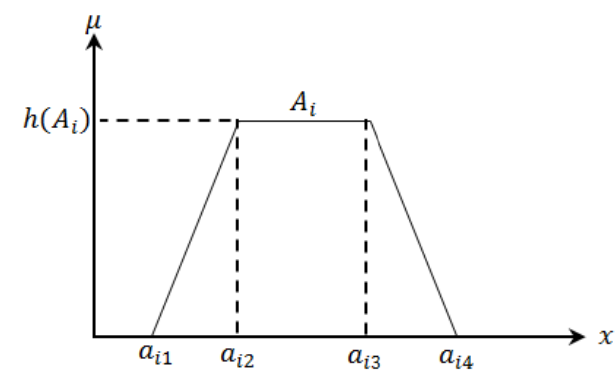

Fig. 1. Membership function (MF) for a trapezoidal T1FS $A_{i}$ 